\title{
CONSTRUCCIÓN DE LA DIVERSIDAD CULTURAL: REPRESENTACIONES Y PRÁCTICAS EN DOCENTES DE LA UNIVERSIDAD VERACRUZANA
}

\author{
María del Rayo Pérez Juárez* \\ Universidad de Veracrúz, México \\ rayperez@uv.mx
}

Recibido: 23/10/2018 Aceptado: 31/03/2019

\begin{abstract}
Resumen
La diversidad cultural se enuncia como una característica y recurso de las sociedades contemporáneas debido a que su reconocimiento permite un tratamiento para potenciar el desarrollo y bienestar colectivo, por lo que hacer un análisis de lo que se comprende como tal es una oportunidad para evidenciar los usos y prácticas que configura. En el texto se presentan las perspectivas de una investigación sobre la diversidad cultural en docentes que participan en la estrategia institucional de profesionalización de la Universidad Veracruzana (UV) ubicada en México para lo que se recurrió a la etnografía educativa y a la teoría de las representaciones sociales. La finalidad de la investigación es contribuir a la visibilización de un concepto polisémico que se traduce en prácticas educativas formadoras de perfiles profesionales que atienden necesidades de sociedades con énfasis creciente en el tratamiento de la diversidad cultural.
\end{abstract}

Palabras clave: Diversidad cultural - Representaciones sociales - Docentes universitarios Etnografía educativa.

\begin{abstract}
Cultural diversity is enunciated as a characteristic and resource of contemporary societies because its recognition allows a treatment to enhance the development and collective well-being, so to make an analysis of what is understood as such is an opportunity to demonstrate the uses and practices that you configure. The text presents the perspectives of a research on cultural diversity in teachers who participate in the institutionalization strategy of the Universidad Veracruzana (UV) located in Mexico for what was used to educational ethnography and the theory of social representations. The purpose of the research is to contribute to the visibility of a polysemic concept that translates into educational practices that shape professional profiles that address the needs of societies with an increasing emphasis on the treatment of cultural diversity.
\end{abstract}

Keywords: Cultural diversity - Social representations - University professors - Educational ethnography.

\section{Introducción}

En el presente texto se presentan las perspectivas de la investigación y tesis de Maestría en Antropología titulada Construcción de la diversidad cultural en docentes de la Universidad Veracruzana: representaciones y prácticas, que sistematizó labúsqueda de aportes para avanzar en la construcción de sociedades plurales, autogestivas y comprensivas desde el escenario educativo. Se investigó para evidenciar la construcción del sentido que comporta el concepto de diversidad cultural en la vivencia de prácticas docentes cotidianas que se inscriben y despliegan en un modelo educativo institucional que se aplica en programas educativos de nivel licenciatura y son formadores de perfiles profesionales llamados a dar respuesta y atención a sociedades diversas con aspiración a ser equitativas e incluyentes. Se considera necesaria esta caracterización debido a su aporte al proceso de exposición de los patrones

* Docente de la Facultad de Antropología de la Universidad Veracruzana, en Veracruz, México. Estudiante del Doctorado en Investigación e Innovación Educativa (DIIE) de la Benemérita Universidad Autónoma de Puebla (BUAP), en Puebla, México 
socioculturales que están implícitos en hábitos y discursos cotidianos y familiares, lo que contribuye a tornarlos incuestionables, vigentes y representativos de la realidad con relación a lo diverso pero que al mismo tiempo hacen parte de la diferencia y la desigualdad social.

Enfocar un concepto tan presente en el escenario educativo como lo es la diversidad cultural, posibilita analizar y comprender la forma en que está constituida para, en primer lugar, reconocer su existencia y posteriormente, las dimensiones socioculturales que permea además de su vivencia con las implicaciones que genera. Así, el reconocimiento de la diversidad cultural como construcción abstracta contribuye a la comprensión de las prácticas concretas en que se traduce (para el caso, en un escenario educativo) que impactan positiva o negativamente en quienes conviven, tal como lo señala Besalú "las estructuras posibilitan unas prácticas y dificultan o impiden otras: marcan un campo de posibilidades" (2002, p.40), lo que insta a afirmar que ni la sociedad ni la cultura, ni las prácticas escolares, funcionan monolítica y acríticamente, como un todo consensuado, sino que sus actores gozan de una autonomía relativa.

\section{Justificación}

Realizar la indagación en el escenario educativo de nivel superior, provee de insumos para identificar las representaciones que constituyen una abstracción (la diversidad cultural), así como sus prácticas en la formación de perfiles de egreso de licenciaturas que se traducirán en desempeños profesionales con los que se busca contribuir al desarrollo plural e inclusivo de la sociedad. Se tiene entonces por pertinente investigar la conformación de un ambiente de aprendizaje específico que descansa en un entendimiento de las relaciones entre docentes, estudiantes y saberes en sus múltiples y variados procesos de aprendizaje y enseñanza que se animan en y a través de modos de vida y de actuar.

Con estos referentes, se entiende que es preciso ahondar en el conocimiento de las interacciones educativas que configuran la propuesta del Modelo Educativo Integral y Flexible (MEIF) perteneciente a la UV cuya intencionalidad sustantiva es ofrecer formación integral a los estudiantes para consolidar ciudadanos éticos y responsables socialmente competentes para interactuar en sociedades complejas y diversas que tienen en sí mismas, procesos competitivos y excluyentes.

\section{Aproximaciones teóricas}

La investigación se abordó desde las teorías de la cultura, específicamente en el paradigma socioestructural de la cultura que se diferencia de los paradigmas descriptivo y el simbólico en el hecho de reconocer a la cultura con desigualdades, contradicciones y asimetrías en el ejercicio del poder, en su carácter conflictivo y desordenado lo que permite entender que la dimensión simbólica de los fenómenos culturales y de las identidades, se insertan en contextos sociales estructurados pero que son procesuales y que implican escenarios espacio-temporales constitutivos de la acción y la interacción que se dan en ellos. En este contexto, el concepto de diversidad cultural se aborda desde una postura crítica que reconoce el carácter intercultural de las sociedades contemporáneas y sus aspiraciones relativas a un estado de bienestar: democracia, equidad, respeto a los derechos humanos y ejercicio de los mismos como marco donde se inscriben instituciones y prácticas que dotan espacios para reflexionar sobre la tensión de lo "universal concreto" y lo "universal abstracto" (Thompson,1998; De la Peña, 2006; Varela,1996).

Concebir la diversidad cultural como una condición de la complejidad de la cultura, propone elementos para reformular la diferencia y la desigualdad, una triada que Dietz y Mateos (2011) proponen como ejes analíticos cuyas combinaciones generan un análisis multidimensional de las identidades y diversidades, lo que proporciona una base conceptual innovadora para reformular el tratamiento o gestión institucional de la diversidad cultural porque interpela a la reflexión de la acción social al situarse en el mundo: esto permite entonces evidenciar los riesgos que advierten algunos teóricos sobre un tratamiento superficial del concepto y su consecuente creación de puntos vacíos de universalidad, desde los cuales se puede apreciar (y despreciar) adecuadamente a los diferentes reafirmando desigualdades y omisiones (Zizek,1998), tal como menciona Teodoro:

la diversidad cultural es insuperable e irresoluble, y no constituye una cuestión de hecho sino una cuestión de derecho; no es el «defecto» sino la "virtud» de la existencia histórico-social del hombre inmersa en un devenir, en movimiento creador y no orden fijo o inamovible (Teodoro, 1996, p.81)

Ahora bien, de la teoría de las representaciones sociales (RS) de Serge Moscovici se utilizó su aporte como unidades que integran el pensamiento con que los sujetos organizan, estructuran y legitiman acciones en su vida cotidiana; se trata de conocimiento primordialmente práctico que posibilita explicar algo y actuar ante ello (Moscovici, 1979). Investigar la configuración de la diversidad cultural desde las RS proporciona un marco de referencia de los actores sociales que fueron los interlocutores, lo que ofrece elementos de análisis sobre sus acciones y abre la posibilidad de producir nuevos comportamientos, gracias a su carácter relacional con otros actores y/o procesos sociales: por ello se dice que las RS son constituidas y constituyentes (Piña y Cuevas, 2004) 


\section{Objetivo}

Como se enuncia, el objetivo de la investigación se planteó investigar las representaciones sobre diversidad cultural en académicos de la Universidad Veracruzana, en el marco de su Modelo Educativo Integral y Flexible y su discurso institucional, para identificar su relevancia en la construcción de prácticas docentes.

La investigación se ubicó en la Universidad Veracruzana, institución mexicana pública y compleja debido a los numerosos y diversos actores que la componen, la cantidad de programas educativos que ofrece y finalmente, su amplia distribución en la geografía del estado de Veracruz, ubicado en el sureste de México. Es esa complejidad institucional la que demanda soluciones innovadoras y pertinentes en sus formas de organización académica y administrativa. Una de esas respuestas para cumplir su función social como formadora de profesionales competentes y actualizados, fue la implementación de una reforma educativa y en consecuencia, de un nuevo modelo educativo que se denomina Modelo Educativo Integral y Flexible (MEIF) el cual enfatiza la formación integral y competente de los estudiantes en un marco de diversidad cultural y equidad, a fin de egresar profesionales con desempeños éticos y propositivos para las sociedades donde se inscriben y desarrollar iniciativas para la atención a necesidades sociales contextualizadas como el combate a la desigualdad social a través de la aplicación del conocimiento y la creación de ciudadanía, por poner un par de ejemplos (Beltrán et al, 1999).

En ese sentido, uno de los actores que cobran protagonismo en la consecucion del objetivo del MEIF, son los docentes, por lo que se recurrió a elaborar un diagnóstico de necesidades de formación docente institucional, con la finalidad de conocer los ámbitos necesarios de atender en cuanto a formación y profesionalización. De los resultados obtenidos del diagnóstico (UV, 2012) resalta el desconocimiento o la polisemia sobre el concepto de diversidad cultural así como el de interculturalidad, ambos declarados institucionalmente como recursos transversales para alcanzar la finalidad del MEIF de consolidar el saber ser y el saber convivir. Acorde a lo anterior, se observó que es en los docentes donde es necesario centrar los esfuerzos de análisis de su práctica para que ésta sea congruente en la promoción y desarrollo de los saberes relacionados con la formación humana, lo que conlleva a que todos los programas educativos reconozcan por un lado, la diversidad cultural presente en sus grupos (y sociedad donde se inscribe el Programa Educativo) y por el otro, la apuesta ética del MEIF de formar en el reconocimiento y gestión de la misma como un recurso para la construcción del conocimiento centrado en valores humanos y sociales, sensible y profunda en cuanto al compromiso social, la conservación y el respeto a la diversidad cultural, la superación personal mediante el autoaprendizaje, el fortalecimiento de la autoestima y el desarrollo de la apreciación por el arte en todas sus manifestaciones (Beltrán et al, 1999).

\section{Aproximaciones Metodológicas}

La investigación se desarrolló articulando las representaciones sociales y la etnografía educativa siguiendo a autores como Banchs (2000), Jiménez (2011) y Bertely (2000) que orientan su estudio en la línea cualitativa, elemento que se tomó en cuenta para la elección del enfoque. Se utilizó entonces la etnografía educativa para hacer el abordaje de los interlocutores en la indagación de las representaciones sobre diversidad cultural desplegada en cuatro fases: la primera constó de la investigación documental que permitió ubicar los referentes sobre la pedagogización de la diversidad cultural y su manifestación en la práctica docente; en la segunda fase se realizó el primer trabajo de campo y ya en la tercera se inició el análisis de la evidencia empírica para hacer ajustes al segundo trabajo de campo, lo que condujo a la cuarta y última fase, donde se analizó la totalidad de la evidencia empírica, se derivaron los resultados y presentaron las conclusiones.

Con respecto a las técnicas e instrumentos, se realizaron entrevistas semi-estructuradas, a profundidad y se realizaron grupos focales para explorar las tres dimensiones de las RS a saber: información, campo de representación y actitudes en este caso, relativas a la diversidad cultural. Los instrumentos utilizados constaron de cartas asociativas, guiones de entrevistas semiestructuradas y entrevistas a profundidad, así como guías de observación y ejercicios producidos en trabajo colaborativo basados en la etnografía educativa tales como visibilizaciones, narraciones colectivas e individuales.

Los interlocutores de la investigación fueron académicos participantes de la estrategia institucional llamada Programa de Formación de Académicos (ProFA) que es la encargada de profesionalizarlos en su dimensión pedagógica y disciplinar a través del desarrollo de cinco competencias (comunicación, autoaprendizaje, planeación, investigación y evaluación) además de cuatro ejes de formación: diseño, tecnología, gestión y sensibilización y tres niveles de desarrollo de los saberes: básico, medio y avanzado que en su intersección creen trayectorias continuas de formación flexibles al ofrecerse en las modalidades presencial, distribuida y virtual. El ProFA se constituye de un catálogo de Experiencias Educativas (EE) diseñadas en apego al MEIF al tiempo que reconoce el poder de acción y elección de los docentes, respeta sus intereses particulares y promueve competencias que contribuyan a consolidar la figura de un docente comprensivo y auténtico, más que uno autoritario y practicante de un rol predeterminado (UV, 1997).

La elección de docentes participantes en el PRoFA corresponde al objetivo de que puedan contrastar su percepción del pasado con la actual sobre la emergencia o transformación que ha tenido el modelo educativo y los recursos que propone como valiosos y pertinentes en sus prácticas, además del conocimiento de sus patrones culturales y el discurso institucional sobre la diversidad cultural. 


\section{Resultados}

La investigación contó con la participación de 83 académicos distribuidos en las tres regiones con mayor participación en el ProFA: Córdoba-Orizaba, Poza Rica-Tuxpan y Xalapa, pertenecientes a varios programas educativos (contaduría, administración, químico industrial, agronomía, gestión intercultural para el desarrollo, trabajo social, pedagogía, lengua francesa, matemáticas, talleres libres de arte, ingeniería química, arquitectura, lengua inglesa, música, derecho, pedagogía, investigaciones forestales, relaciones internacionales y enfermería), de todas las áreas académicas de la UV: ciencias de la salud, humanidades, artes, multidisciplinaria, económica administrativa y área técnica.

El promedio de edad de los docentes interlocutores es de 44.5 años (siendo el más joven de 27 años y el mayor de 62) y con un tiempo de dedicación a la docencia de 12.75 años, donde el menos experimentado en funciones de docencia cuenta un año de práctica en contraste con quien sumó 26 , aunque en todos los casos se trata de docentes que cuentan con una trayectoria formativa en el ámbito de la profesionalización de su práctica. De este modo, tienen un marco de referencia que les posibilita expresar las condiciones en que realizan su mediación tanto como los requerimientos que la configuran, de modo que comprenden la labor docente como un entramado de prácticas más complejo que la denominada transmisión del conocimiento o el dar clase; esto se manifiesta en sus dichos cuyas expresiones encuentran recurrencia en lo que teóricos como Contreras (2011) refiere como la competencia profesional, que es la dimensión que manifiesta la conciencia de sentido en las prácticas al dotarla de un carácter integrador que se ve interpelado por un contexto sociocultural e institucional (expuesto por el modelo educativo) y un horizonte amplio que parte de la experiencia vital que descansa (o aspira) a la realización de formas alternativas de construir sociedades y modos de relación.

La investigación rastreó las tres dimensiones de las RS en el concepto de diversidad cultural y derivado de ello es que fue posible hacer una caracterización de la misma en dos estadios: de aprendiz principiante y de aprendiz avanzado. Se denominó con el término aprendiz (aunque sea en dos niveles diferenciados) a los tipos, por considerar que son aproximaciones procesuales y en constante modificación, de modo que sugieren su dinamismo y complejización de acuerdo a las condiciones contextuales, los recursos de los que dispongan los docentes, los ritmos de aprendizaje y la creación de la necesidad por reflexionar y acercarse al tema de la diversidad en cada docente; es decir, se mantiene la posición de seguir aprendiendo.

El aprendiz principiante es aquél que se apega a un concepto de cultura e identidad como entidades discretas que no aceptan discordancias significativas en su seno y que requiere de modelos pedagógicos y prácticas concretas que les permitan conservar, restaurar, normar y nivelar sus rasgos distintivos. Esta caracterización implica que los docentes privilegian la concepción de grupos de aprendizaje homogéneos en el sentido más tradicional del término: los convoca una intención de formación y es la que se privilegia a las demás características de los estudiantes. Tal concepción está sostenida en la suposición de que los estudiantes forman parte de una cultura y una sociedad bien compacta y representada en un canon o norma: se trata de identidades colectivas que se adscriben y corresponden a un determinado tipo de sociedad que no muestra grandes desniveles en sus características y que es posible denominar como una unidad, donde por otro lado, tales muestras de diversidad son secundarias o no representativas de la unidad sino que pueden asociarse al ámbito de la vida privada o cuya significatividad es restringida o nula.

En lo que toca al aprendiz avanzado, denota una tensión entre la concepción de grupos de aprendizaje homogéneos o más bien estandarizados y la experiencia de su diversidad inherente, lo que se sostiene en una aproximación más flexible con relación a las identidades sociales y culturales de los estudiantes. En consecuencia, se trata de una construcción dinámica que permite características de diferencia entre los estudiantes si bien ésta se expresa en características más o menos evidentes y reconocibles para los docentes: se trata de rasgos distintivos que son identificados, si bien no se sabe siempre y del todo el cómo establecer relaciones con ellos.

Esta construcción de la diversidad cultural (DC) en los docentes evidenció las superposiciones, desviaciones, agregados, contradicciones y tensiones entre todo un repertorio de elecciones y condiciones sobre la misma, lo que permite que se refleje en una gama amplia de prácticas como una suerte de espiral que atraviesa las dimensiones de la docencia propuestas por Contreras (2011) y que son competencia profesional, obligación moral y compromiso con la comunidad.

De la evidencia empírica emanaron datos relevantes que permitieron ubicar la tensión entre lo fáctico y lo discursivo de la diversidad cultural en los interlocutores y sus prácticas, ya que mientras en algunas respuestas relativas a su desempeño docente se posicionaban como dialógicos, flexibles a la transformación sociocultural y respetuosos de la diversidad, en otros cuestionamientos tocantes con las actitudes, se reconocían con una postura más bien conservadora o incluso prescriptiva con relación a lo diverso.

La conciencia de estar en un ámbito de actuación con un claro compromiso político y ético, se relaciona con las significaciones bajo las que se desarrolla la práctica educativa docente lo que representa una forma de intervenir en las condiciones socioculturales que le competen a la educación, ya que desde la práctica docente y la formación profesional se está desempeñando un papel en la educación de los sujetos sociales que tiene algún efecto en su vida (incluida la vida futura) y tiene también una pretensión de colaborar en la construcción de prácticas con sentido social, tendientes al favorecimiento o resistencia y/o reproducción y legitimación de la injusticia y la desigualdad, por ejemplo.

La investigación arrojó elementos para problematizar el papel del docente ante la heterogeneidad de 
sus estudiantes y sus formas de relación con ellos donde se consolidaron como aportes significativos la honestidad y el ejercicio metacognitivo de los interlocutores que permitieron visibilizar las formas en que los toman decisiones, realizan elecciones y ejercitan prácticas y modos de relación que pueden acercarse o no a un discurso o narrativa imperante e institucional. Finalmente, el MEIF es el referente sobre el que se ejercen modos concretos de experiencias que a su vez, recrean significados compartidos o discordantes del contexto sociocultural en que están sucediendo y que en lo referente al reconocimiento de las características del modelo, incluida la diversidad cultural, los docentes refieren un conocimiento estereotípico, general y superficial a pesar de contar con experiencia acumulada y participación activa en las estrategias institucionales de profesionalización.

Los conceptos nodales en la propuesta del MEIF que suponen el viraje al anterior modelo educativo, quedan desdibujados en la ambigüedad, desconocimiento e imprecisión desde las respuestas de los interlocutores, quienes en su mayoría, no logran ubicar las directrices, estrategias ni documentos donde se declaran los objetivos y propuestas del MEIF, así como tampoco reconocen de manera inmediata las dimensiones del mismo, sus ejes, las áreas de formación o el enfoque metodológico que orienta y evalúa los diseños curriculares de sus planes y programas de estudio, de modo que el concepto de diversidad cultural (que está mucho menos presente de modo explícito en los documentos institucionales) tampoco goza de gran reconocimiento ni caracterización lo que genera diferencias y discordancias significativas en las comprensiones que los docentes hacen del propio MEIF y sus recursos, lo que en la expresión de prácticas, representa una gran variedad y en algunos casos, son una reiteración de exclusiones y/o omisiones que inhiben u obstaculizan el logro del modelo educativo.

No obstante, los resultados emanados y acotados al escenario de esta investigación, hacen que resulte sugerente esta polisemia y polivalencia en la toma de postura de los docentes con relación a la diversidad cultural y sus expresiones ya que exige primero un reconocimiento de la misma y una elaboración de sus características y modos de animarse en prácticas, las cuáles se desarrollan y tienen presencia en los espacio donde se realiza la mediación pedagógica del docente y al final de cuentas, el acto educativo. Por eso, las políticas y prácticas para la diversidad en educación reclaman precisión acerca de lo que significan y nombran para aclarar ambigüedades e imprecisiones que en muchos casos, dotan de una sobrecarga semántica (con sus adjudicaciones) a ciertos conceptos, lo que obstaculiza o inutiliza su comprensiión y motivan alón y discus desgano y desconcierto en las prácticas.

Las funciones atribuidas a los docentes para que atiendan esferas cada vez más difusas y ambiguas de la realidad social, se traduce muchas veces en una "racionalidad instrumental" que les permiten tener una práctica que se concreta a seguir las directrices que suponen una protección ante la responsabilidad, de modo que así, hay sólo procedimientos correctos, lo que conlleva a la realización de prácticas del modo adecuado y alineado con la definición institucional (plasmada en el modelo educativo, en el ideario de la práctica, etc.), todo esto en contradicción con la misión encomendada a la educación superior que plantea como sus finalidades más sentidas aquellas formas de formación para consolidar un desempeño profesional crítico que responda a las necesidades y retos de sociedades plurales (Gimeno, 2005).

\section{Conclusiones}

Esta investigación es un ejercicio de interseccionalidad que se enfocó en el reconocimiento de la diversidad cultural desde una interpelación a los docentes en sus procesos de construcción de la misma, ya que implica el hecho de ahondar en el conocimiento de sus estudiantes, pero supone también un reconocimiento de sus propios procesos de aprendizaje, enseñanza e interacciones, así como de las dificultades para hacer tales profundizaciones. Es decir, se evidencia que el ejercicio profesional de la docencia requiere de un posicionamiento en la realidad estrechamente anclado en la cotidianidad y la especificidad de sus demandas, necesidades y requisitos.

Como apunta Contreras (2011) la reflexión crítica y metacognitiva es un proceso de pensamiento con el claro propósito de "definirse" ante las situaciones que se presentan en la labor que se realiza. Como muestra la investigación, esta reflexividad no es instrumental sino que analiza el tipo de vinculación con la acción que busca establecer, bajo qué relaciones sociales realizarla, a qué intereses sirve y qué construcción social apoya. Es por eso que la metacognición crítica ayuda a analizar las condiciones sociales y de historia en que se han formado los modos de entender y valorar la práctica docente y su relación con la diversidad cultural, lo que problematiza su carácter político desde que insta a reconstruir los procesos de formación y construcción social que sostienen ciertas representaciones de la educación así como a estudiar sus contradicciones y tensiones.

Hundir la reflexión en las representaciones de los sujetos sociales y comprender su relación con sus prácticas requiere de un pensamiento individual y colectivo que oriente la colaboración y aproveche la diversidad de la condición humana, como una posibilidad para reconstruir, en este caso, estructuras educativas y sociales con prácticas cada vez más sólidas (reflexivas e intencionadas) y pertinentes.

\section{Referencias bibliográficas}

- Banchs, M. (2000). "Aproximaciones procesuales y estructurales al estudio de las representaciones sociales". Paper on Social Representations Texte sur les represéntations sociales. (9) 3.1-3.15.

- Beltrán, J. et al. (1999). Nuevo Modelo Educativo para la Universidad Veracruzana. Lineamientos para el nivel de licenciatura. Propuesta. Universidad Veracruzana, Xalapa, México

- Bertely, M. (2000). "Investigación etnográfica en escuelas y salones de clase: la entrada al campo". En: M. 
Bertely: Conociendo nuestras escuelas: un acercamiento etnográfico a la cultura escolar, pp. 43-62. Paidós, México.

- $\quad$ Besalú, X. (2002). Diversidad cultural y educación. Editorial Síntesis, Madrid, España.

- $\quad$ Contreras, J. (2011). La autonomía del profesorado. Editorial Morata, España.

- De la Peña, G. (2006). "Articulación y desarticulación de las culturas". En David Sobrevilla. (Ed.) Filosofía de la cultura. Editorial Trotta, Madrid, España.

- Dietz, G. y L. Mateos (2011). Interculturalidad y educación intercultural en México. Un análisis de los discursos nacionales e internacionales en su impacto en los modelos educativos mexicanos. Secretaría de Educación Pública. Coordinación General de Educación Intercultural y Bilingüe, México.

- Gimeno, J. (2002). Educar y convivir en la cultura global. (2da edición). Morata, España.

- $\quad$ (2005). La educación que aún es posible. Morata, España.

- Jiménez, Y. (2009). "Indeterminación conceptual en las prácticas educativas interculturales: los conceptos de cultura e identidad a examen". En: Maya Lorena Pérez Ruiz, Laura Raquel Valladares de la Cruz y Margarita Zárate (eds.) Estados plurales: los retos de la diversidad y la diferencia, pp. 349-373. UAM-I y Juan Pablos Editor, México.

- Loughlin, C.E. y J.H.Suina (1995). El ambiente de aprendizaje: diseño y organización. Editorial Morata, España.

- Moscovici, S. (1979). El psicoanálisis su imagen y su público. Edit. Huemul, Buenos Aires.

- Pérez, M. (2016). Construcción de la diversidad cultural en docentes de la Universidad Veracruzana: representaciones y prácticas. Tesis de Maestría. Universidad Veracruzana. Xalapa, Veracruz, México.

- Piña, J. y Cuevas, Y. (2004). "La teoría de las representaciones sociales. Su uso en la investigación educativa en México". Perfiles Educativos, XXVI Tercera época, pp. 102-124.

- Teodoro, M. (1996). "Muchas culturas. Sobre el problema filosófico y práctico de la diversidad cultural". En: Ursula Klesing-Rempel (comp.) \& Astrid Knoop (coord.). Lo propio y lo ajeno. Interculturalidad y sociedad multicultural, 19-47. Plaza y Valdés- Asociación Alemana para la Educación de Adultos Instituto de la Cooperación Internacional, México.

- Thompson, J. (1998). Ideología y cultura moderna. Teoría Crítica social en la era de la comunicación de masas. UAM-X-DCSyH, México.

- Universidad Veracruzana (1997). Documento Propuesta del Programa de Formación de Académicos. Inédito, México.

- $\quad$ Documento interno de trabajo

- Varela, Roberto. (1996). "Cultura política". En: Héctor Tejera (coord.), Antropología política. Enfoques contemporáneos, INAH/Plaza y Valdés Eds.. México

- Zizek Z. (1998). "Multiculturalismo o la lógica cultural del Capitalismo multinacional", en Estudios culturales. Reflexiones sobre el multiculturalismo. Paidós, Buenos Aires. 